\title{
Designing Gold Nanoparticle-Ensembles as Surface Enhanced Raman Scattering Tags inside Human Retinal Cells
}

\author{
Sanda Boca, ${ }^{1}$ Dumitrita Rugina, ${ }^{2}$ Adela Pintea, ${ }^{2}$ Nicolae Leopold, ${ }^{3}$ and Simion Astilean ${ }^{1}$ \\ ${ }^{1}$ Nanobiophotonics and Laser Microspectroscopy Center, Institute for Interdisciplinary Research in Bio-Nano-Sciences and \\ Faculty of Physics, Babes-Bolyai University, Treboniu Laurian 42, 400271 Cluj-Napoca, Romania \\ ${ }^{2}$ Department of Biochemistry, University of Agricultural Sciences and Veterinary Medicine, Manastur 3-5, 400372 Cluj-Napoca, \\ Romania \\ ${ }^{3}$ Faculty of Physics, Babes-Bolyai University, Kogalniceanu 1, 400084 Cluj-Napoca, Romania
}

Correspondence should be addressed to Sanda Boca, sanda.boca@phys.ubbcluj.ro

Received 10 January 2012; Accepted 15 February 2012

Academic Editor: Brian M. Cullum

Copyright (C) 2012 Sanda Boca et al. This is an open access article distributed under the Creative Commons Attribution License, which permits unrestricted use, distribution, and reproduction in any medium, provided the original work is properly cited.

Apart from the traditional development of surface-enhanced raman scattering (SERS) substrates for ultrasensitive spectroscopic analysis, an increasing interest is given nowadays to the design of the so-called SERS nanotags which integrate multiple SERS applications into single plasmonic nanoparticles. The fabrication of SERS tags is still a challenging task due to the complicated fabrication process. Typically, SERS tags are hybrid nanoconstructs consisting in a unique plasmonic nanoobject encoded with specific reporter molecules and enveloped in a protective shell that provides both biocompatibility and targeting function. Herein, we produce effective SERS tags consisting in small aggregates of gold nanoparticles (mainly dimers and trimers) which are captured from solution and then transferred into cells to perform as individual plasmonic nanostructures. Actually the small aggregates formed under controlled conditions are stabilized in solution by interlocking into a polymeric envelope made of thiol-modified poly(ethylene) glycol (PEG-SH). No further encoding operation is necessary in our case since part of ascorbic acid used as reducing agent remains attached in the interparticle junctions, providing persistent and strong SERS signal when the fabricated tags are internalized by human retinal cells. Our studies demonstrate a promising potential of new SERS-active nanoparticles to serve as effective reporters for biomedical tracing and imaging.

\section{Introduction}

The development of various strategies for the preparation of new optical labels as probes for detection and imaging has gained tremendous interest in the past years [1-4]. Among the investigation techniques, Raman spectroscopy presents real advantages due to the unambiguous identity of the encoded probe and the availability of a large pallet of reporter molecules [5]. Although Raman spectroscopy is reliable, the poor efficiency of Raman scattering (the crosssection is equal to $10^{-30} \mathrm{~cm}^{2}$ molecule $\mathrm{e}^{-1}$ ) has limited its use in biomedical applications. Therefore the enhancement of the vibrational signal of the encoded molecule is required to improve the detection speed and sensitivity. In this context, surface-enhanced Raman scattering (SERS) spectroscopy proved to be a unique solution for the amplification of the vibrational signal by factors of $10^{6}$ and even as high as $10^{14}$ $10^{15}$ in some cases $[6,7]$. With such a large enhancement of Raman scattering, the use of SERS for imaging applications is an attractive alternative to fluorescence. Unlike fluorescent labels as are organic fluorophores and quantum dots, SERS-encoded probes, composed of a layer of Raman reporter molecules bound onto a metal nanoparticle, have the advantage of being resistant to photobleaching. This is due to the quenching of fluorescence excited states by the metal surface and to the short lifetime of Raman virtual energy states [8]. Furthermore, using SERS technique, different Raman reporters can be simultaneously excited with a single light source of choice (such as near-infrared light for human tissue) to achieve quantitative multiplexed detection [9]. These advantages confer to such SERS labels wide applicability in biomedical systems. 
As stated above, the general strategy for designing SERSencoded nanoparticles or SERS nanotags involves the attachment of one or multiple organic dyes as signature reporters onto a metal enhancer most usefully supplied in the form of a gold or silver nanoparticle, which is further encapsulated by a polymeric, biomolecular, or a glass shell for protection against aggregation and biocompatibilization. The literature is recently abundant with such encoded probes as for example the polymer-stabilized gold nanoparticles reported by Merican et al. [10], protein-capped composite organicinorganic nanoparticles described by Su et al. [11], silica embedded core-shell nanoparticles reported by Mulvaney et al. [12], or silica-encapsulated $\mathrm{Au} / \mathrm{Ag}$ nanoshells described by Küstner et al. [13]. More recently an increased attention was given to the implementation of improved substrates for SERS nanoprobes such as anisotropic or multibranched nanoparticles, which exhibit strong plasmon resonances close to the NIR window of biological transparency and, particularly, high electromagnetic field localized at their protrusions [14-16]. Our group succeeded to demonstrate the efficiency of such dye-encoded multi-branched flowershaped nanoparticles as SERS tags inside living cells [17]. The SERS nano-tags were designed using malachite green oxalate and basic fuchsin as Raman-active molecules and protected against aggregation with thiol-modified poly(ethylene) glycol polymer. As-prepared nanotags were found to be highly stable, to be SERS detectable inside living cells under $633 \mathrm{~nm}$ laser excitation, and to present low in vitro toxicity when tested inside the cytosol of a line of epithelial cells from human retina.

In this paper, we extend our research and present a new class of nano-tags consisting of gold nanoparticle ensembles (i.e., dimers, trimers, and small aggregates of spherical particles) with real potential to act as SERS-active tags inside living cells. The particles were fabricated by an alternative method of the commonly Turkevich-Frens synthesis, by the use of ascorbic acid as reducer of the gold salt [18]. Notably, this class of nanoparticles does not require any supplementary operation of encoding since ascorbic acid remains attached in between nanoparticles and give a persistent and strong SERS signal when nano-tags are internalized by cells. Therefore we expect that our tags present higher biocompatibility than SERS tags marked with potentially toxic, Raman-active organic dyes. Moreover, our fabrication strategy for the assembly of AuNPs differs from most of the reported techniques which involve crosslinking molecules, like alkanethiols $[19,20]$, surfactants [21], specific proteins [22], and oligonucleotides [23] and most often destabilizing species like salt or ethanol [24], which might further interfere with the intrinsic particles signal. Herein thiol-modified PEG, a nontoxic, hydrophilic polymer, commonly used to improve particles stability, biocompatibility, and their systemic retention [25], is used for multiple purposes: to link the particles between them and stabilize the formed nanoensembles. To the best of our knowledge, no other reports demonstrate SERS-active tags with high and reproducible intrinsic signal coming from the reducing agent used in particle synthesis. The signal reproducibility in simulated body fluid salted solution, and in vitro measurements on human retinal cells highlight the potential of such SERSactive nanoparticles to serve as biomedical imaging tools.

\section{Experimental Section}

2.1. Chemicals and Materials. Hydrogen tetrachloroaurate (III) trihydrate $\left(\mathrm{HAuCl}_{4} \cdot 3 \mathrm{H}_{2} \mathrm{O}\right)$ was purchased from Sigma-Aldrich (Germany). Analytical-grade L-ascorbic acid $\left(\mathrm{C}_{6} \mathrm{H}_{8} \mathrm{O}_{6}\right)$ was obtained from Reactivul (Romania). $\alpha$ Methoxy- $\omega$-mercapto poly(ethylene glycol) (mPEG-SH) of molecular weight $5 \mathrm{kDa}$ was obtained from Iris biotech GmbH (Germany). GIBCO Dulbecco's Modified Eagle's Medium (DMEM) was purchased from Invitrogen (Carlsbad, California, USA). Fetal bovine serum, penicillin, streptomycin, amphotericin B, and 3-(4,5-dimethylthiazol-2-yl)2,5-diphenyltetrazolium bromide (MTT) were purchased from Sigma (St. Louis, USA). Dimethyl sulfoxide, sodium pyruvate, and all the others chemicals used were of analytical grade and supplied from Merck (Germany). The other reagents used through the experiments were analytical grade and used without further purification. Distilled deionized water $\left(\mathrm{ddH}_{2} \mathrm{O}\right)$ was used in all aqueous solutions and rinsing procedures.

\subsection{Preparation of PEG-Protected Gold Nanoparticle Ensem-} bles. All glassware used in the synthesis was cleaned in a bath of freshly prepared aqua regia solution $(3: 1(\mathrm{v} / \mathrm{v})$ $\mathrm{HNO}_{3}: \mathrm{HCl}$ ) and rinsed thoroughly prior to use. The colloidal gold nanoparticles were synthesized based on a method described elsewhere in detail [18]. Briefly, individual nanoparticles were produced by stirring $10 \mathrm{~mL}$ of a solution of $0.5 \cdot 10^{-3} \mathrm{M}$ tetrachloroauric acid $\left(\mathrm{HAuCl}_{4}\right)$ at room temperature for several minutes, followed by the addition of a proper volume of a freshly prepared ascorbic acid $\left(7.5 \cdot 10^{-3} \mathrm{M}\right)$ solution. Herein, tetrachloroauric acid trihydrated $\left(\mathrm{HAuCl}_{4} \cdot 3 \mathrm{H}_{2} \mathrm{O}\right)$ has a role of nanoparticle initiator while L-ascorbic acid is used as reducer of the gold salt. When the two reactive solutions were mixed, the yellow pale dispersion rapidly turned colorless, dark blue and finally pinkish red. This color transition indicated the reduction of the $\mathrm{Au}^{3+}$ ions to gold atoms which undergo nucleation and form the colloidal nanoparticles by diffusional growth.

For designing gold nanoparticles aggregates, increasing volumes of $10^{-6} \mathrm{M}$ mPEG-SH solution $(20 \mu \mathrm{L}, 50 \mu \mathrm{L}$, and $100 \mu \mathrm{L}$ ) were added to the colloidal solution by dripping and let to sit for several minutes. The ratio between the colloid and polymer solution and the incubation time were carefully chosen so that small aggregates (two to several nanoparticles) would be first formed. Excess polymer stabilized the formed ensembles of nanoparticles. Before being incubated in living cells, the as-prepared probes were purified by centrifugation at low speed.

2.3. Equipments Used for Samples Characterization. Optical extinction spectra were measured with a Jasco V-670 spectrophotometer over a spectral range between 400 and $1000 \mathrm{~nm}$ and a spectral resolution of $2 \mathrm{~nm}$. The mean diameter of gold nanoparticles was determined by transmission 
electron microscopy (TEM) imaging using a JEOL model JEM1010 microscope. SERS spectra of gold nanoparticles and PEG-capped nanoensembles in aqueous solution were recorded using for excitation NIR $(785 \mathrm{~nm})$ laser line from a diode laser using a portable Raman spectrophotometer (R-3000CN from Raman Systems) with $1 \mathrm{~cm}^{-1}$ spectral resolution and an integration time of $10 \mathrm{sec}$. SERS spectra of nanoparticle ensembles inside living cells were recorded using the $633 \mathrm{~nm}$ laser line of a He-Ne laser. The $633 \mathrm{~nm} \mathrm{He}-$ Ne laser delivered a power of $3 \mathrm{~mW}$ through a 100x objective $(\mathrm{NA}=0.9)$ of a confocal Raman microscope alpha 300R from WITEC.

2.4. Cell Culture and MTT Viability Assay. Human adult retinal pigment epithelial cells D407 were maintained in Dulbecco's modified eagle medium supplemented with $10 \%$ fetal bovine serum, $1 \mathrm{mM}$ sodium pyruvate, $100 \mathrm{U} / \mathrm{mL}$ penicillin, $100 \mu \mathrm{g} / \mathrm{mL}$ streptomycin, and $2.5 \mu \mathrm{g} / \mathrm{mL}$ amphotericin $\mathrm{B}$, at $37^{\circ} \mathrm{C}, 5 \% \mathrm{CO}_{2}$, and $95 \%$ relative humidity. The cells were seeded in 96 well-plates at a concentration of $1 \times 10^{4}$. After reaching $90 \%$ confluence, growth medium was removed and the cells were incubated for $24 \mathrm{~h}$ with uncoated nanoparticles (GNPs) and PEGylated gold ensembles (PEG-GENs) by varying the colloidal concentration between $0.3 \times 10^{-11} \mathrm{M}$ and $6 \times 10^{-11} \mathrm{M}$. The tetrazolium salt was used to quantify living metabolically active cells, based upon the principle that MTT (3-(4,5-dimethylthiazol-2-yl)-2,5-diphenyltetrazolium bromide) is metabolized by mitochondrial dehydrogenase to form a formazan dye. Thereby the end product was measured at a wavelength of $550 \mathrm{~nm}$ with background wavelength at $690 \mathrm{~nm}$, with a microplate plate reader HT BioTek Synergy (BioTek Instruments, USA). Briefly, the cells were washed with PBS, and $200 \mu \mathrm{L}$ MTT solutions in HBSS buffer were added to each well. After $2 \mathrm{~h}$ of incubation the MTT reagent was removed and the formazan particles were solubilized with $200 \mu \mathrm{L}$ DMSO. Viability was expressed as the percentage of actively proliferative cells, and subsequently a relationship between viability and treatment concentrations was plotted as shown in Figure 6. Statistical analysis was conducted using the Tukey multiple comparison test of Graph Pad Prism version 5.00. Data are presented as means and standard errors of the mean (SEM). (*Significant $P<0.05$, **very significant $P<0.01$.)

\section{Results and Discussion}

3.1. Characterization of Gold Nanoparticle Ensembles and Probing Their SERS Activity. The normalized extinction spectrum of as-prepared gold nanoparticles, the building blocks of gold nanoparticle ensembles, is illustrated in Figure 1 (spectrum a) and presents a plasmon resonance band at $540 \mathrm{~nm}$. When few $\mu \mathrm{L}$ of mPEG-SH polymer were added to the colloidal solution, the plasmon peak red-shifted by $3 \mathrm{~nm}$, which is consistent with an increase of the refractive index surrounding the nanoparticles, due to the polymeric capping (magnified image, Figure 1). Together with the observed shift, a slight broadening of the plasmon band occurred and a secondary, smaller-intensity band appeared,

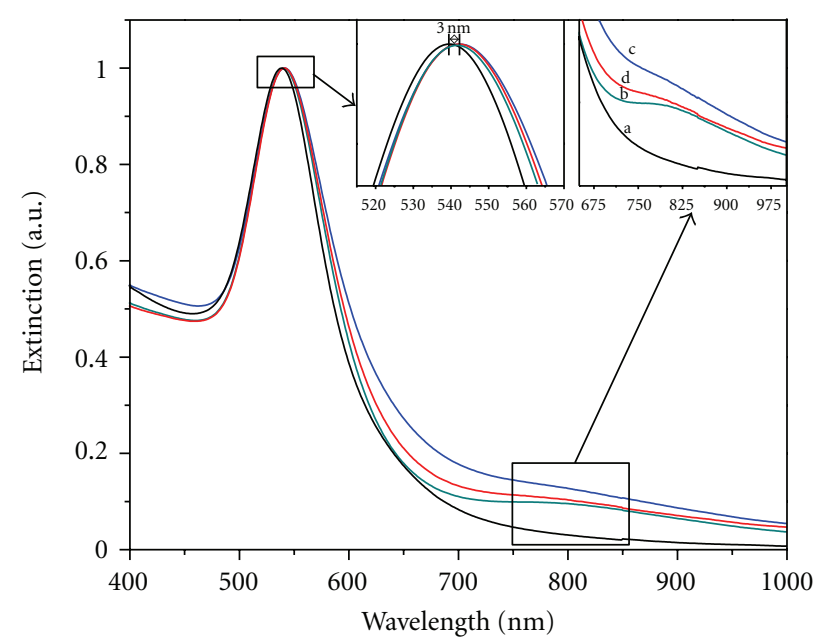

FIGURE 1: Normalized extinction spectra of individual gold nanoparticles (spectrum a) and of PEGylated gold nano-ensembles obtained by the addition of: $20 \mu \mathrm{L}$ (spectrum b), $50 \mu \mathrm{L}$ (spectrum c) and $100 \mu \mathrm{L}$ (spectrum d), of mPEG-SH polymer into the colloidal solution.

situated at about $800 \mathrm{~nm}$ which indicates the formation of few nanoparticle aggregates in the colloidal solution. Further increasing the ratio between the polymer and the colloidal solution determined the broadening of both main $(543 \mathrm{~nm})$ and secondary $(800 \mathrm{~nm})$ plasmonic band (spectra $c$ and $d$ in Figure 1). Afterward, no more red shift of the main plasmonic band was observed, demonstrating that individual PEGylated nanoparticles were completely capped by the polymer. When the maximum concentration of the polymer was added to the colloidal nanoparticles, the band corresponding to ensembles of nanoparticles featured only negligible red shift, indicating that further aggregation was inhibited and the formed PEG-GENs remained in that aggregation state thereafter. The small intensity drop of the plasmon band observed for this later sample is only caused by the dilution and not by the sedimentation of the aggregates.

The particles morphology and the formation of particleensembles were subsequently confirmed by TEM measurements. According to Figure 2(a), as-prepared colloids contain mainly individual, spherical nanoparticles, having a mean diameter of $50 \mathrm{~nm}$. Colloids with added PEGs presented also dimers, trimers, and small clusters (Figures 2(b), $2(\mathrm{c})$, and $2(\mathrm{~d})$ ) which are surrounded by a faint uniform layer of approximately $3 \mathrm{~nm}$ of PEG polymeric chains. The clusters of up to five nanoparticles (less than $300 \mathrm{~nm}$ ) are suitable for intracellular detection due to their small sizes, capable to penetrate through the membrane of a living cell. Moreover, such nanoparticle arrangements in small aggregates are responsible for the strong electromagnetic fields (hot spots) in which trapped Raman-active molecules have scattering signals increased up to $10^{14}$.

The general strategy to fabricate SERS-active nano-tags consists in selecting a molecule with a strong Raman signal and binding the selected molecule to a noble metal nanoparticle of suitable optical response [26]. Usually polymers are further involved in that design, in order to increase both 


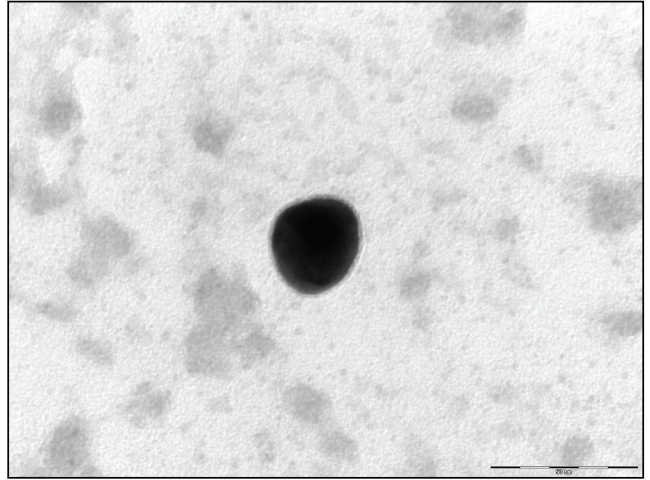

(a)

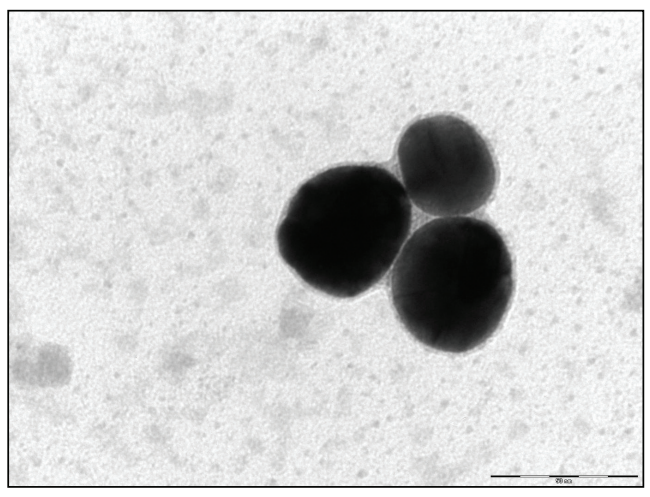

(c)

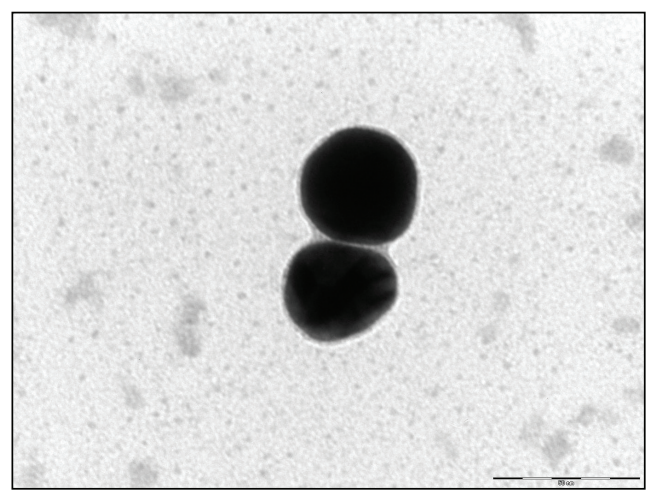

(b)

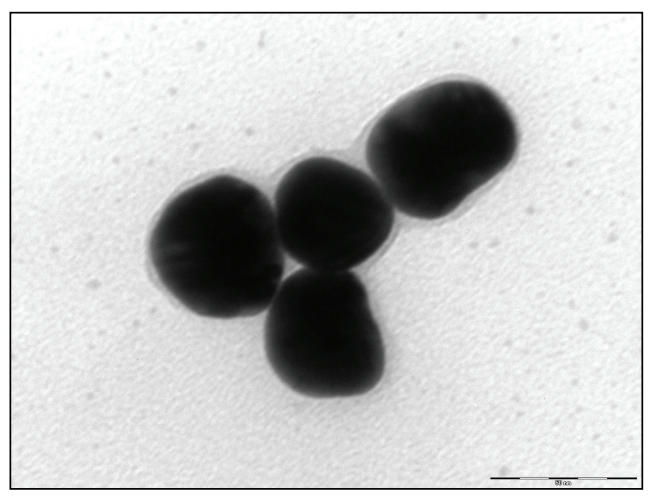

(d)

Figure 2: TEM images of single (a), dimer (b), trimer (c), and a small cluster of spherical gold nanoparticles. Scale bar $50 \mathrm{~nm}$.

nanoparticle stability and biocompatibility. For isolated solid spherical Au or Ag nanoparticles with smooth surfaces, the SERS enhancements are reported to be pretty low, with the enhancement factors on the order of $10^{3}$ [27]. Therefore, nanoparticles with bumps and cavities like stars, meatballlike or flower-shaped, were preferred because the localized near-field enhancements on the tip of nanoscale bumps or inside tiny cavities provide SERS enhancements that are significantly larger than those achievable on a solid spherical particle with a smooth surface. Nearly adjacent nanoparticles with nanoscale gaps have been shown to serve as efficient and extremely sensitive SERS substrates [28,29]. One of the main attributes of such nanoprobes lies in the electronic coupling between the particles which leads to many new properties [30]. For example, this coupling effect has been widely accepted as the cause of the so-called "hot spots" or areas of highly intense electromagnetic fields upon plasmonic excitation in which the efficiency of surface-enhanced Raman scattering (SERS) is dramatically increased [31].

To probe the SERS activity of PEG-GENs, we excited the nano-ensembles in aqueous solution with $785 \mathrm{~nm}$ NIR laser line. This excitation wavelength superposes the characteristic plasmon band of the PEG-GENs and also provides maximum light penetrability for further application in biological media such are cells and tissues [32]. We also investigated on which extent the concentration of PEG polymer affects the SERS fingerprint of such nano-ensembles, and we correlated the obtained spectra with the PEG-GENs size and morphology.

SERS spectra of the formed nano-ensembles obtained with increasing concentrations of PEG-SH are illustrated in Figure 3, and the band assignments can be found in Table 1. SERS spectrum of nanospheres (spectrum b) in aqueous solution and Raman spectrum of pure L-ascorbic acid (spectrum a) were also recorded to serve for comparison. The SERS spectra of PEG-GENs (spectra c, d, e) present characteristic vibrational bands of the ascorbic acid molecules. No signal from the PEG polymeric layer is observed which is in agreement with other reports from the literature [33]. Meaningful variations of bands intensities are observed between the three probes, which we correlate with the aggregation degree of individual gold nanoparticles, hence the size and morphology of the nanoaggregates. PEGGENs prepared with the highest concentration of PEG present the highest SERS signal. We speculate that part of the thiolated polymeric chains links the particles between them when added to the colloidal solution, contributing to the formation of the nano-aggregates, while the polymer in excess stabilizes the formed nano-ensembles, and therefore the SERS signal is conserved.

Few band positions differences from one sample to another are also detected, mostly at higher wave numbers 


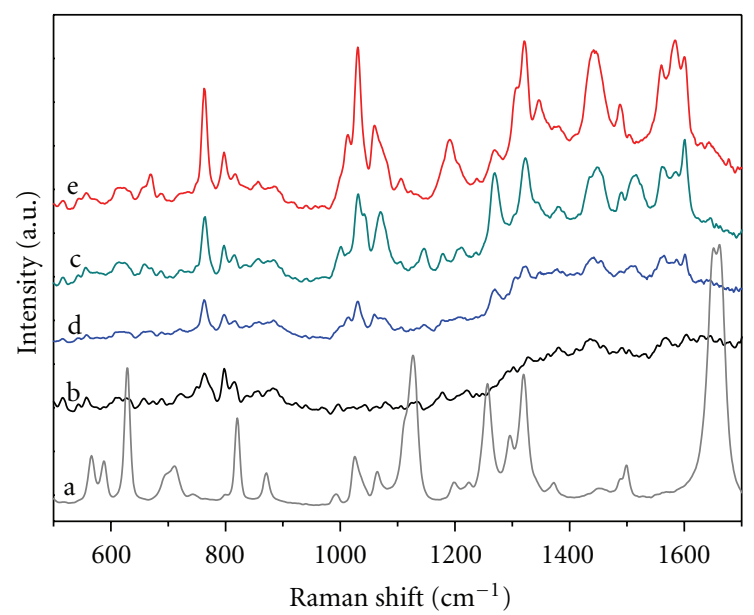

Figure 3: Raman spectrum of solid pure L-ascorbic acid (scaled by factor 0.25 )(spectrum a). SERS spectrum of individual gold nanospheres in solution recorded under NIR excitation (spectrum b). SERS spectra of PEG-GENs obtaine with increasing volumes: $20 \mu \mathrm{L}$ (spectrum c), $50 \mu \mathrm{L}$ (spectrum d), and $100 \mu \mathrm{L}$ (spectrum e) mPEG-SH, recorded under NIR excitation.

$\left(1200-1800 \mathrm{~cm}^{-1}\right)$, which arise from different ascorbic acid molecular orientations on the particles surface. A variation of cluster geometry is also responsible for different arrangements of the adsorbed molecules in the formed gaps between particles. The ratios between the intensities of specific vibrational bands such as $1030 \mathrm{~cm}^{-1}(\mathrm{C}-\mathrm{O}-\mathrm{H}$ bend) and $1061 \mathrm{~cm}^{-1}\left(\mathrm{C}-\mathrm{O}-\mathrm{C}\right.$ str), $1322 \mathrm{~cm}^{-1}(\mathrm{C}-\mathrm{H}$ bend) and $1270 \mathrm{~cm}^{-1}, 1488 \mathrm{~cm}^{-1}$ (C-H bend) and $1504 \mathrm{~cm}^{-1}$ (C$\mathrm{H}$ bend) modify and a triplet band situated at $1584 \mathrm{~cm}^{-1}$ appears instead of the intense doublet at $1656 \mathrm{~cm}^{-1}(\mathrm{C}=\mathrm{C}$ str in ring) in the Raman spectrum of pure ascorbic acid. An early study regarding the Raman spectra of ascorbic acid and its related compounds reported by Edsall and Sagall assigns this band to the ascorbate anions of the dissociated ascorbic acid [34]. Herein, this extra band at $1584 \mathrm{~cm}^{-1}$, together with the disappearance of the vibrational bands characteristic to the $\mathrm{C}=\mathrm{C}$ str in the lactone ring after $1650 \mathrm{~cm}^{-1}$ lead us to infer that the SERS spectra of PEGGENs are assigned in fact to the doubly oxidized, more stable form of the ascorbic acid, dehydroascorbic acid. This stable form arises as a consequence of the ascorbic acid degradation by oxidization in the presence of metal ions, during the nanoparticles synthesis. These molecules are further adsorbed onto nanoparticles surface and then captured in the gaps between nanoparticles through the influence of the polymer, giving rise to strong SERS signal when excited by NIR laser light. Therefore, we demonstrated that we can design PEG-nano-ensembles that are capable to give intrinsic SERS signal in solution without necessitating the addition of supplementary Raman active molecules, potentially toxic to bioenvironments. We mention that the SERS signal is conserved after sample purification while no signal was obtained from individual, as prepared, uncoated nanoparticles in the colloidal suspension (spectrum $b$ in Figure 3).
TABLE 1: Raman and SERS bands of L-ascorbic acid and their vibrational assignment

\begin{tabular}{|c|c|c|}
\hline Raman & SERS & Vibrational assignment $\mathrm{t}^{\mathrm{a}}$ \\
\hline $743 w$ & $763 \mathrm{~s}$ & O-H out of plane def. \\
\hline $798 \mathrm{sh}$ & 800 & $\mathrm{O}-\mathrm{H}$ out of plane def. \\
\hline $820 s$ & 819 & $\mathrm{C}=\mathrm{C}$ str in ring \\
\hline $87 \mathrm{~m}$ & 883 & $\mathrm{C}=\mathrm{C}$ str in ring \\
\hline $993 w$ & 1012 & $\mathrm{C}-\mathrm{H}$ bend; $\mathrm{O}-\mathrm{H}$ bend \\
\hline $1025 \mathrm{~m}$ & 1030 & $\mathrm{C}-\mathrm{O}-\mathrm{H}$ bend \\
\hline $1064 w$ & 1061 & $\mathrm{C}-\mathrm{O}-\mathrm{C}$ str. \\
\hline $1128 v s$ & 1190 & $\mathrm{C}-\mathrm{O}-\mathrm{C}$ str. \\
\hline $1198 w$ & 1211 & $\mathrm{C}-\mathrm{C}(=\mathrm{O})-\mathrm{O}$ str. \\
\hline \multicolumn{3}{|l|}{$1225 \mathrm{w}$} \\
\hline $1256 \mathrm{~s}$ & & $\mathrm{C}-\mathrm{O}-\mathrm{C}$ bend $+\mathrm{tw}$ \\
\hline $1295 \mathrm{~m}$ & 1270 & \\
\hline $1319 s$ & 1322 & C-H bend \\
\hline $1372 w$ & 1380 & $\mathrm{C}-\mathrm{O}-\mathrm{H}$ bend $+\mathrm{CH}_{2} \mathrm{w}$ \\
\hline $1451 w$ & $1441 \mathrm{~s}$ & $\mathrm{C}-\mathrm{H}$ bend $+\mathrm{CH}_{2} \mathrm{sc}$ \\
\hline $1489 w$ & $1488 \mathrm{~m}$ & $\mathrm{C}-\mathrm{H}$ bend \\
\hline $1498 \mathrm{~m}$ & $1504 w$ & $\mathrm{C}-\mathrm{H}$ bend \\
\hline 1656s split & 1584 triplet & $\mathrm{C}=\mathrm{C}$ str in ring \\
\hline
\end{tabular}

${ }^{a}$ References [34-36].

3.2. Chemical Stability of PEG-GENs in Simulated Biological Fluid. One important requirement for any probe to be used inside living organisms is that it has to retain its properties in salted media as is the cellular medium. Moreover, the investigated probe must provide efficient and distinctive readout (e.g., SERS signal) in these specific conditions. To test the chemical stability of the prepared ensembles and evaluate the protection degree of the PEG shell, we first measured optical extinction spectra of PEG-GENs after the addition of a high molarity solution of sodium chloride, known to induce particle aggregation in the case of unprotected gold colloids [37]. The obtained results were compared with that of uncoated nanoparticles.

Figure 4 illustrates the extinction spectra of uncoated, as-prepared nanoparticles (black curves) and of PEG-GENs obtained with various amounts of PEGs (colored curves) in the absence (full lines) and in the presence (dashed lines) of chloride ions. The appearance of the plasmon resonance band in the case of uncoated nanoparticles was visibly altered after the addition of salt. The peak position shifted to longer wavelengths by several $\mathrm{nm}$, and a secondary band at about $900 \mathrm{~nm}$ rapidly developed, demonstrating that, in the absence of PEG protection, the gold nanoparticles rapidly aggregate. Monitored, these aggregated nanoparticles turned into irreversible sediments within hours.

PEG-GENs obtained with low concentration of PEG polymer showed similar behavior with that of unprotected particles. The ratio between the intensity of main plasmonic band at $543 \mathrm{~nm}$ and that of the band specific to nanoclusters 


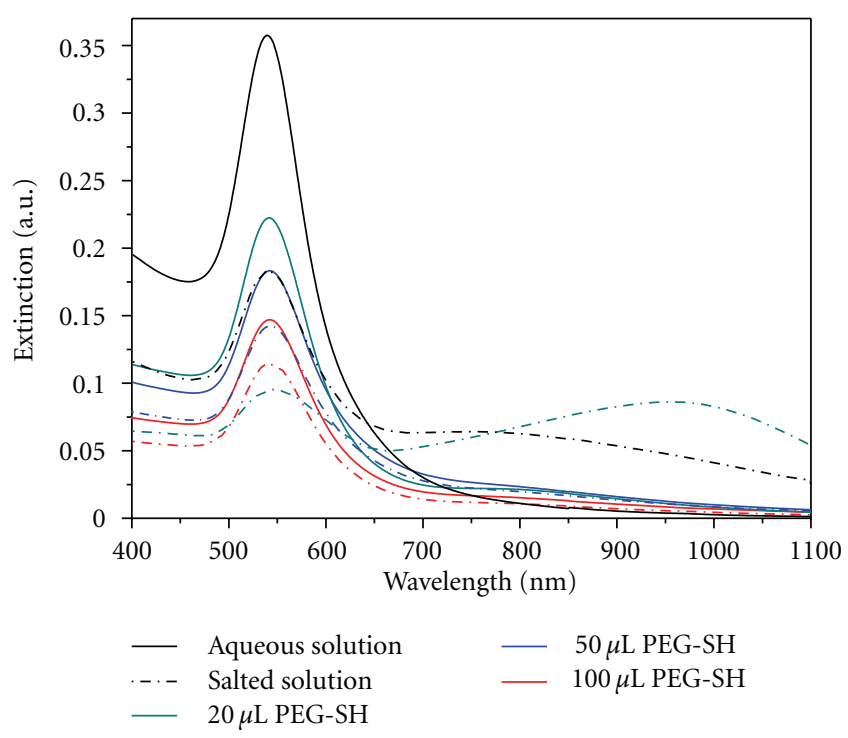

FIgURE 4: Extinction spectra of gold nanospheres ((GNSs) black line) and of PEGylated gold nanoensembles ((PEG-GENs) colored lines) recorded in the absence (thick lines) and in the presence (dotted lines) of $1 \mathrm{M} \mathrm{NaCl}$ solution.

rapidly decreased, due to the formation of more and larger aggregates in the colloidal solution. An increase of PEG polymer concentration contributed to a visible stabilization of the formed GENs, as can be observed from the presented spectra (blue and red curves in Figure 4), which maintained a constant position and band shape, even in the presence of the supplementary aggregation agent. The small drop observed in the extinction intensity is attributed to the sample dilution, caused by the addition of the salted solution. Although slight precipitation might occur after the sample is being kept for a longer time, the sediment can be easily redispersed by sonication.

We further tested the SERS signal stability in the presence of $\mathrm{Cl}^{-}$ions of the PEG-GENs (Figures 5(b) and 5(c)). Uncoated, individual GNPs (Figure 5(a)) were tested in similar conditions. We found that for both uncoated nanoparticles and for nanoensembles prepared with low concentration of PEG the SERS signal increased after the addition of salt. We corroborate this result with an overaggregation of the particles, which lack sufficient PEG protection. Although aggregation substantially enhances SERS [38], from an imaging or sensing application standpoint uncontrolled aggregation is undesirable since the resultant SERS signal fluctuates. Moreover large aggregates hardly can penetrate cellular membrane, impeding any use of the particles for in vivo applications. In the case of PEG-GENs prepared with higher polymeric concentration, the SERS signal remains almost steady after the addition of sodium chloride (Figure 5(d)). The minor intensity changes might be caused by the modification of the reporter molecules orientation on the gold surface [39]. In our design, the thiol-PEG layer maintains the ascorbate molecules adsorbed onto nanoparticle surface, by steric stabilization. Moreover, the result demonstrates that PEG not only protects the formed ensembles from further aggregation but also controls their aggregation degree by inhibiting the cohesion between particles.

3.3. Viability of D407 Cells Incubated with Nanoparticle Ensembles. The effect of nano-tags toward cell viability was tested using MTT assay. The obtained results were compared with that of uncoated nanoparticles and that of the supernatant solution. The cell survival in the presence of the selected probes is plotted in Figure 6. For all the incubated samples, cells' viability and proliferation are highly dependent on particle concentration. Cells incubated with PEG-GEN have a high (80\%) survival rate for nanoparticles concentration maintained up to $2.4 \times 10^{-11} \mathrm{M}$, after which a steady decrease of cell viability is observed. Significant toxicity for the cells (less than $40 \%$ from the total number of cells remain viable after $24 \mathrm{~h}$ ) appears only for the highest nanoparticle concentration $\left(6 \times 10^{-11} \mathrm{M}\right)$. At this high concentration bare GNPs gave better survival rate while supernatant was proved to be more cytotoxic than the prepared PEG GENs nano-tags. From the class of hydrophilic polymers used as stabilizing agents for various types of nanoparticles, PEG polymer was generally proved to be biologically inert [40]. Moreover, PEG and PEG-derivatives were largely involved in detoxifying nanoparticles such are for example CTAB-toxic gold nanorods towards use in cellular environment [41, 42]. Bearing this in mind, the increased biocompatibility of naked nanoparticles compared to that of SERS nanotags might appear intriguing. To explain this, we infer a different mechanism of delivery through cells for the two types of nanoprobes. Naked nanoparticles lacking the protection of the PEG coating would aggregate in salted cellular medium forming big clusters, which are unable to penetrate the cellular membrane and therefore would not be delivered through cells. PEG-stabilized nanoensembles on the other hand would be delivered to the cellular compartments in greater amount causing slight toxicity but only at high nanoparticle concentration. The effect of supernatant solution containing only unbound polymer and unreduced gold ions also sustains the above hypothesis.

3.4. PEG-GENs as SERS Tags inside Living Cells. Surfaceenhanced Raman scattering is a highly sensitive spectroscopic technique that has been imposed as a convenient and attractive alternative to fluorescence for detection and imaging applications inside living cells [43]. Despite the unique advantages offered by SERS, the huge amount of molecules found in cell cytoplasm is often an impediment towards the extraction of relevant biological information. Moreover, lack of signal reproducibility might occur due to a common tendency of nanoparticles to aggregate in buffer solutions or serum. Although substantial enhancement of SERS is obtained if nanoparticles aggregate, from an imaging and detection standpoint, such phenomenon is hardly desirable, since aggregation cannot be controlled and the resultant SERS signal fluctuates. Also big aggregates hardly can enter the cellular membrane. Recently, more sensitive nano-tags were developed by encapsulating organic dyes as signature reporter molecules between gold nanoparticles and 


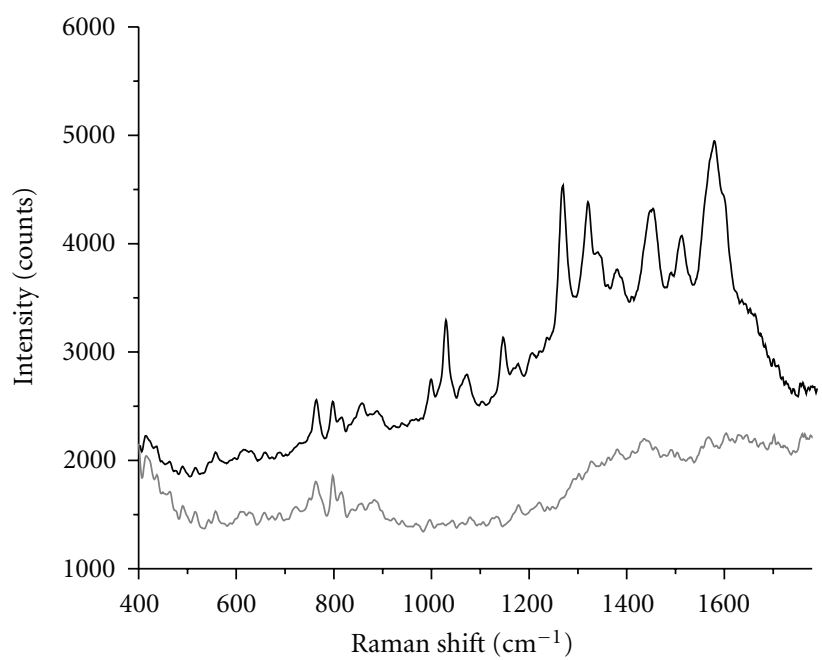

(a)

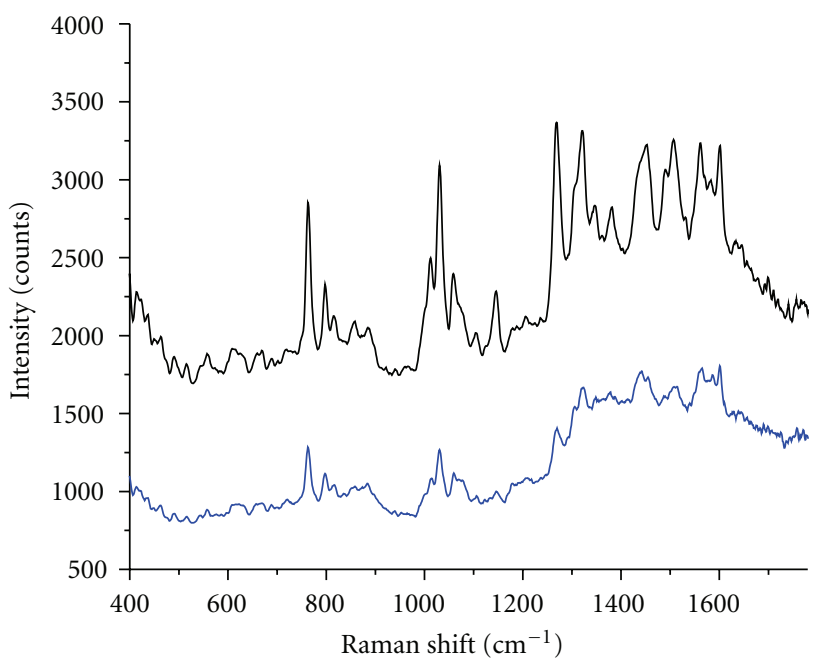

(c)

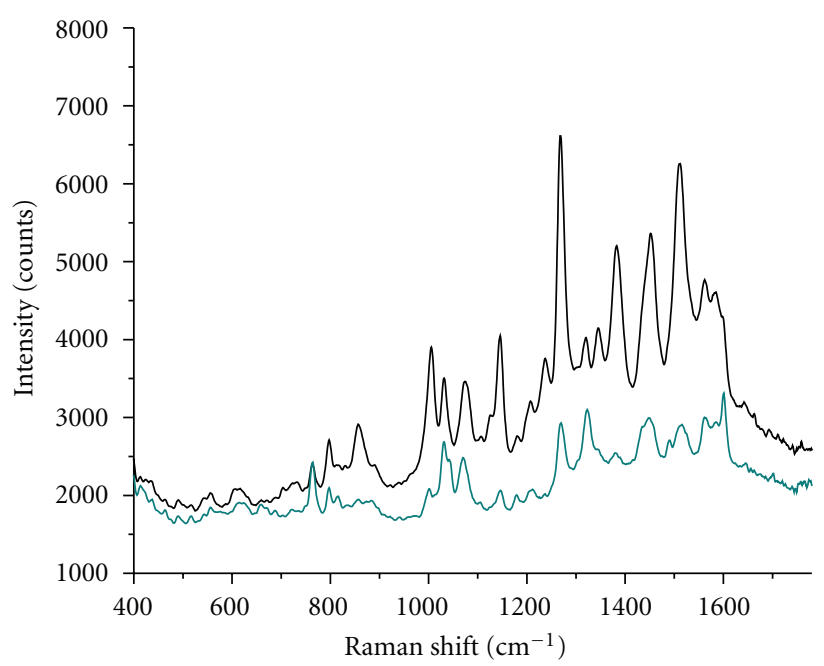

(b)

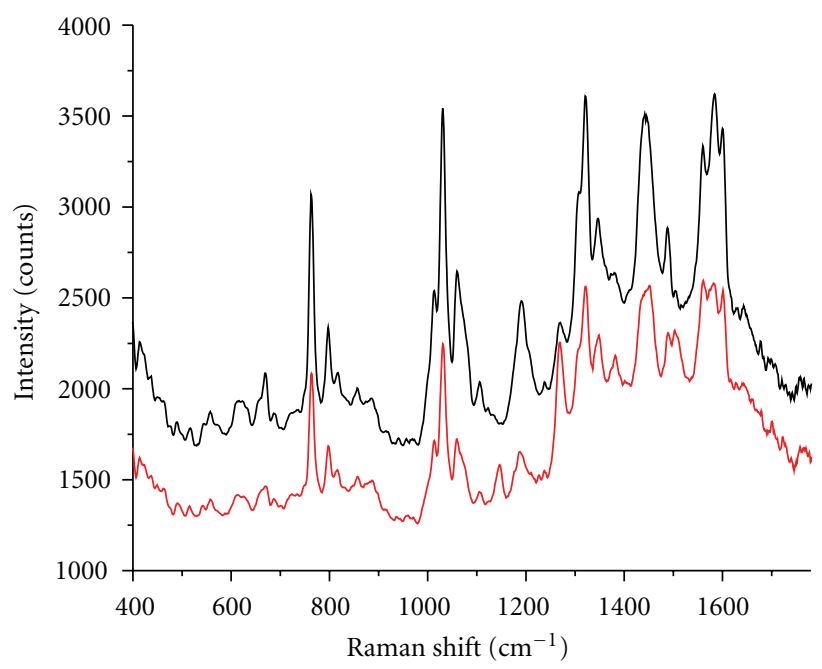

(d)

FIGURE 5: Raman spectrum of gold nanospheres (a) and SERS spectra of PEG-GENs prepared with increasing volumes: $20 \mu \mathrm{L}$ (b), $50 \mu \mathrm{L}$ (c), and $100 \mu \mathrm{L}$ (d) of mPEG-SH polymer, recorded in solution, under NIR excitation. Black spectra are taken in the presence of $1 \mathrm{M}$ salted solution while colored spectra represent the spectra of the nano-probes before the addition of salt.

a polymeric shell for biocompatibilization and stabilization. Such active tags are able to trigger specific phenomena inside cells or can be bound to desired cellular organelles [44, 45].

To determine whether SERS spectra can be acquired from ascorbate-tagged, PEG-protected GENs buried inside cells, we incubated D407 cells with the particles for 24 hours and recorded SERS spectra of tagged nano-probes inside cells. The first optical evaluations of the cellular samples indicate an intracytoplasmic and endoplasmatic reticulum distribution of the probes (Figure $7(\mathrm{a})$ ). The predominant distribution pattern is nearby the nucleus, without triggering any particles in the nuclear region.

SERS detection of the nano-probes inside living cells followed the microscopic visualisation. SERS spectra which highly resemble the Raman-encoded particles signature in solution were obtained at positions in the cells where the particles were located as can be seen in Figure 7(b) (spectrum c).
Apart from vibrational bands specific to ascorbic acid reporter molecules, intense SERS bands (the bands marked by asterisks in spectrum c) are also observed in the case of PEGylated GENs inside cells at $672 \mathrm{~cm}^{-1}, 848 \mathrm{~cm}^{-1}$, and in the $1150-1250 \mathrm{~cm}^{-1}$ fingerprint region. These bands can be attributed to phospholipids and cellular proteins $(\mathrm{C}-\mathrm{C}$ skeletal stretch in proteins and amide III/ $\beta$-sheet resp.) which intercalate the polymeric chains and adsorb onto some of the nanoparticles surface $[43,46]$. Moreover, specific bands $\left(1004 \mathrm{~cm}^{-1}\right.$ phenylalanine: ring breathing, $1320 \mathrm{~cm}^{-1}$ adenine/proteins: ring stretching/twisting $\left(\mathrm{CH}_{2}\right.$, $\left.\mathrm{CH}_{3}\right), 1491 \mathrm{~cm}^{-1}$ proteins: deformation $\left.\left(\mathrm{CH}_{2}, \mathrm{CH}_{3}\right)\right)$ superimpose those of the reporter molecules. Therefore, we can fairly assert that the recorded spectra are composite spectra combining the response of encoding ascorbate molecules and cellular constituents. No Raman signal could be obtained from cells in the absence of nanoparticles (spectrum a in 


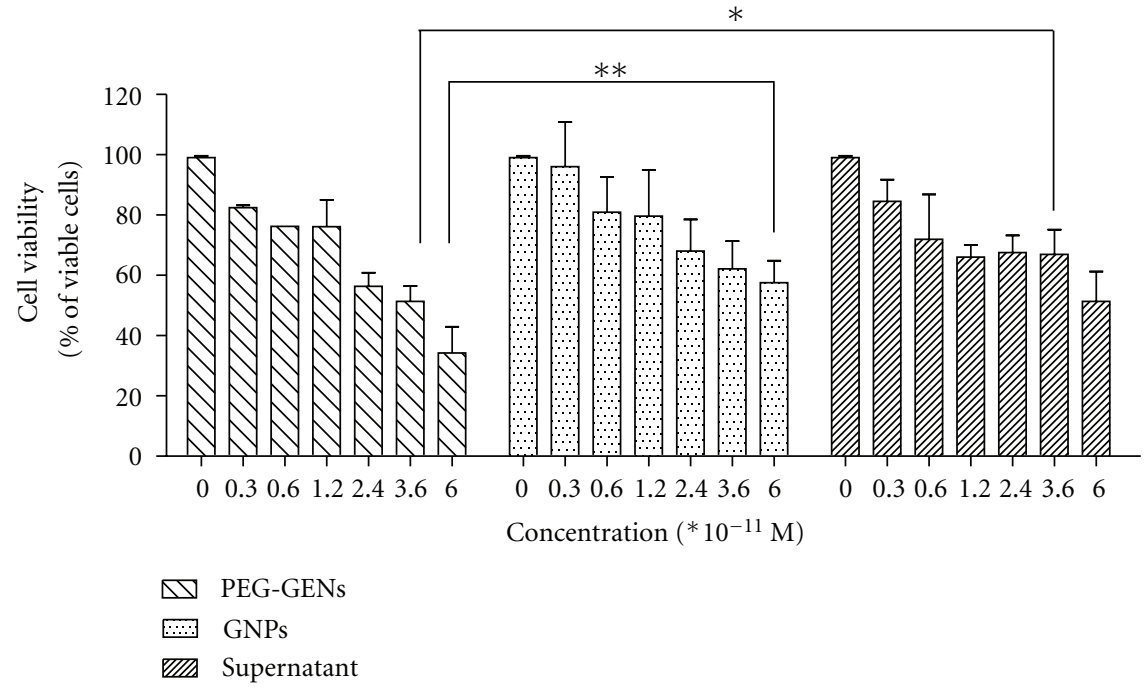

FIGURE 6: MTT test of epithelial cells from human retina (D407 cells viability (\%)) treated with various concentrations of PEG-GENs and GNPs. The effect of supernatant onto cell viability is also presented. Data are presented as means and standard errors of the mean (SEM): * significant $P<0.05,{ }^{* *}$ very significant $P<0.01$.

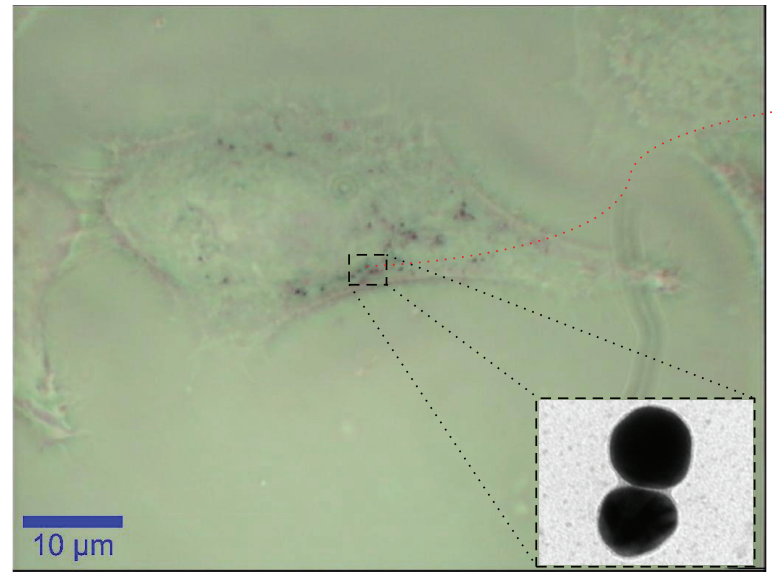

(a)

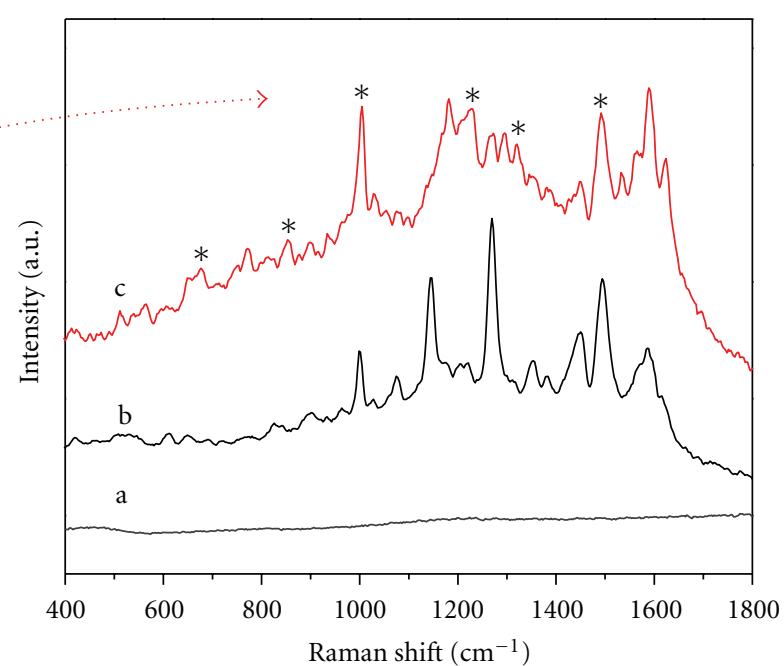

(b)

FIGURE 7: Optical microscopy image of a D407 cell loaded with ascorbate-encoded PEG-GENs (a). SERS spectra of as prepared PEG-GENs in aqueous solution (spectrum b in (b)) and inside the cell (spectrum c in (b)) recorded under 633 nm laser line excitation. The asterisks above spectrum c indicate vibrational modes that correspond to cellular components. Spectrum a in (b) represents the Raman spectrum of an individual cell in the absence of nanoparticles, as control.

Figure 7(b)). The above results demonstrate that our PEGGENs with intrinsic signal from ascorbic acid drug molecules have the advantage of providing readable SERS response inside cells without involving the aid of any supplementary molecules, potentially toxic to cells.

\section{Conclusions}

This work presents a new class of SERS-active tags consisting of PEG-protected gold nanoparticles ensembles (PEGGENs) encoded with trace of ascorbic acid which was previously used as reducing agent in the nanoparticle synthesis.
We found that PEG-SH polymer does not only stabilize individual nanoparticles in solution but can also capture, interlock, and stabilize a few number of nanoparticles as dimers, trimers, and small aggregates. The fabrication of SERS tags presents the advantage of easy, single-step encoding procedure by exploiting the presence of ascorbic acid in solution during nanoparticle formation. The PEGGENs retain both the spectroscopic identity of ascorbic acid and optical activity of aggregates, both in saline solution and inside living cells, without interfering too much with cellular functions. Notably, the polymer coating provides not only good biocompatibility with biological media but exhibits 
chemical availability for implementing specific targeting functions.

\section{Acknowledgments}

This work was supported by the CNCS-UEFISCDI, Project number PNII-ID_PCCE_129/2008. The authors are grateful to Dr. Lucian Barbu Tudoran for TEM measurements.

\section{References}

[1] D. C. Pregibon, M. Toner, and P. S. Doyle, "Multifunctional encoded particles for high-throughput biomolecule analysis," Science, vol. 315, no. 5817, pp. 1393-1396, 2007.

[2] J. Yang, M. P. Sena, and X. Gao, "Quantum dot-encoded fluorescent beads for biodetection and imaging," Reviews in Fluorescence, vol. 2007, pp. 139-156, 2009.

[3] K. B. Cederquist, S. L. Dean, and C. D. Keating, "Encoded anisotropic particles for multiplexed bioanalysis," Wiley Interdisciplinary Reviews: Nanomedicine and Nanobiotechnology, vol. 2, no. 6, pp. 578-600, 2010.

[4] K. T. Thurn, E. M. B. Brown, A. Wu et al., "Nanoparticles for applications in cellular imaging," Nanoscale Research Letters, vol. 2, no. 9, pp. 430-441, 2007.

[5] R. A. Alvarez-Puebla and L. M. Liz-Marzán, "SERS-based diagnosis and biodetection," Small, vol. 6, no. 5, pp. 604-610, 2010.

[6] R. F. Aroca, Surface-Enhanced Vibrational Spectroscopy, John Wiley \& Sons, New York, NY, USA, 2006.

[7] M. Moskovits, "Surface-enhanced Raman spectroscopy: a brief retrospective," Journal of Raman Spectroscopy, vol. 36, no. 6-7, pp. 485-496, 2005.

[8] X. Qian, X. H. Peng, D. O. Ansari et al., "In vivo tumor targeting and spectroscopic detection with surface-enhanced Raman nanoparticle tags," Nature Biotechnology, vol. 26, no. 1, pp. 83-90, 2008.

[9] A. Matschulat, D. Drescher, and J. Kneipp, "Surface-enhanced Raman scattering hybrid nanoprobe multiplexing and imaging in biological systems," ACS Nano, vol. 4, no. 6, pp. 32593269, 2010.

[10] Z. Merican, T. L. Schiller, C. J. Hawker, P. M. Fredericks, and I. Blakey, "Self-assembly and encoding of polymer-stabilized gold nanoparticles with surface-enhanced Raman reporter molecules," Langmuir, vol. 23, no. 21, pp. 10539-10545, 2007.

[11] X. Su, J. Zhang, L. Sun et al., "Composite Organic-Inorganic Nanoparticles (COINs) with chemically encoded optical signatures," Nano Letters, vol. 5, no. 1, pp. 49-54, 2005.

[12] S. P. Mulvaney, M. D. Musick, C. D. Keating, and M. J. Natan, "Glass-coated, analyte-tagged nanoparticles: a new tagging system based on detection with surface-enhanced Raman scattering," Langmuir, vol. 19, no. 11, pp. 4784-4790, 2003.

[13] B. Küstner, M. Gellner, M. Schütz et al., "SERS labels for red laser excitation: silica-encapsulated SAMs on tunable gold/silver nanoshells," Angewandte Chemie-International Edition, vol. 48, no. 11, pp. 1950-1953, 2009.

[14] T. Wang, X. Hu, and S. Dong, "Surfactantless synthesis of multiple shapes of gold nanostructures and their shapedependent SERS spectroscopy," Journal of Physical Chemistry B, vol. 110, no. 34, pp. 16930-16936, 2006.

[15] C. Hrelescu, T. K. Sau, A. L. Rogach, F. Jäckel, and J. Feldmann, "Single gold nanostars enhance Raman scattering," Applied Physics Letters, vol. 94, no. 15, Article ID 153113, 2009.
[16] G. H. Jeong, Y. W. Lee, M. Kim, and S. W. Han, "Highyield synthesis of multi-branched gold nanoparticles and their surface-enhanced Raman scattering properties," Journal of Colloid and Interface Science, vol. 329, no. 1, pp. 97-102, 2009.

[17] S. Boca, D. Rugina, A. Pintea, L. Barbu-Tudoran, and S. Astilean, "Flower-shaped gold nanoparticles: synthesis, characterization and their application as SERS-active tags inside living cells," Nanotechnology, vol. 22, no. 5, Article ID 055702, 2011.

[18] S. C. Boca, M. Potara, F. Toderas, O. Stephan, P. L. Baldeck, and S. Astilean, "Uptake and biological effects of chitosan-capped gold nanoparticles on Chinese Hamster Ovary cells," Materials Science and Engineering C, vol. 31, no. 2, pp. 184-189, 2011.

[19] M. G. Bellino, E. J. Calvo, and G. Gordillo, "Adsorption kinetics of charged thiols on gold nanoparticles," Physical Chemistry Chemical Physics, vol. 6, no. 2, pp. 424-428, 2004.

[20] A. Hofmann, P. Schmiel, B. Stein, and C. Graf, "Controlled formation of gold nanoparticle dimers using multivalent thiol ligands," Langmuir, vol. 27, no. 24, pp. 15165-15175, 2011.

[21] L. Sun, D. Zhao, M. Ding et al., "Controllable synthesis of silver nanoparticle aggregates for surface-enhanced raman scattering studies," Journal of Physical Chemistry C, vol. 115, no. 33, pp. 16295-16304, 2011.

[22] M. Ringler, T. A. Klar, A. Schwemer et al., "Moving nanoparticles with Raman scattering," Nano Letters, vol. 7, no. 9, pp. 2753-2757, 2007.

[23] D. B. Lukatsky and D. Frenkel, "Surface and bulk dissolution properties, and selectivity of DNA-linked nanoparticle assemblies," The Journal of chemical physics, vol. 122, no. 21, Article ID 214904, 11 pages, 2005.

[24] X. Han, J. Goebl, Z. Lu, and Y. Yin, "Role of salt in the spontaneous assembly of charged gold nanoparticles in ethanol," Langmuir, vol. 27, no. 9, pp. 5282-5289, 2011.

[25] G. F. Paciotti, D. G. I. Kingston, and L. Tamarkin, "Colloidal gold nanoparticles: a novel nanoparticle platform for developing multifunctional tumor-targeted drug delivery vectors," Drug Development Research, vol. 67, no. 1, pp. 47-54, 2006.

[26] J. P. Nolan and D. S. Sebba, "Surface-enhanced raman scattering (SERS) cytometry," Methods in Cell Biology, vol. 102, pp. 515-532, 2011.

[27] Z. Zhu, T. Zhu, and Z. Liu, "Raman scattering enhancement contributed from individual gold nanoparticles and interparticle coupling," Nanotechnology, vol. 15, no. 3, pp. 357-364, 2004.

[28] J. P. Camden, J. A. Dieringer, Y. Wang et al., "Probing the structure of single-molecule surface-enhanced Raman scattering hot spots," Journal of the American Chemical Society, vol. 130, no. 38, pp. 12616-12617, 2008.

[29] H. Ko, S. Singamaneni, and V. V. Tsukruk, "Nanostructured surfaces and assemblies as SERS media," Small, vol. 4, no. 10, pp. 1576-1599, 2008.

[30] P. K. Jain and M. A. El-Sayed, "Noble metal nanoparticle Pairs: effect of medium for enhanced nanosensing," Nano Letters, vol. 8, no. 12, pp. 4347-4352, 2008.

[31] I. A. Larmour, E. A. Argueta, K. Faulds, and D. Graham, "Design consideration for surface-enhanced (resonance) Raman scattering nanotag cores," Journal of Physical Chemistry C, vol. 116, no. 4, pp. 2677-2682, 2012.

[32] X. Huang, I. H. El-Sayed, W. Qian, and M. A. El-Sayed, "Cancer cell imaging and photothermal therapy in the nearinfrared region by using gold nanorods," Journal of the American Chemical Society, vol. 128, no. 6, pp. 2115-2120, 2006. 
[33] L. Rodríguez-Lorenzo, R. A. Álvarez-Puebla, F. J.G. De Abajo, and L. M. Liz-Marzán, "Surface enhanced Raman scattering using star-shaped gold colloidal nanoparticles," Journal of Physical Chemistry C, vol. 114, no. 16, pp. 7336-7340, 2010.

[34] J. T. Edsall and E. L. Sagall, "Raman spectra of l-ascorbic acid, tetronic acid and related compounds," Journal of the American Chemical Society, vol. 65, no. 7, pp. 1312-1316, 1943.

[35] C. Yohannan Panicker, H. Tresa Varghese, and D. Philip, "FTIR, FT-Raman and SERS spectra of vitamin C," Spectrochimica Acta-Part A, vol. 65, no. 3-4, pp. 802-804, 2006.

[36] H. Yang and J. Irudayaraj, "Rapid determination of vitamin C by NIR, MIR and FT-Raman techniques," Journal of Pharmacy and Pharmacology, vol. 54, no. 9, pp. 1247-1255, 2002.

[37] C. Mangeney, F. Ferrage, I. Aujard et al., "Synthesis and properties of water-soluble gold colloids covalently derivatized with neutral polymer monolayers," Journal of the American Chemical Society, vol. 124, no. 20, pp. 5811-5821, 2002.

[38] N. Leopold, J. R. Baena, M. Bolboacă, O. Cozar, W. Kiefer, and B. Lendl, "Raman, IR, and surface-enhanced Raman spectroscopy of papaverine: an automated setup for in situ synthesis of the silver substrate and recording of the SER spectra," Vibrational Spectroscopy, vol. 36, no. 1, pp. 47-55, 2004.

[39] J. D. Jiang, E. Burstein, and H. Kobayashi, "Resonant raman scattering by crystal-violet molecules adsorbed on a smooth gold surface: evidence for a charge-transfer excitation," Physical Review Letters, vol. 57, no. 14, pp. 1793-1796, 1986.

[40] A. W. Basit, F. Podczeck, J. M. Newton, W. A. Waddington, P. J. Ell, and L. F. Lacey, "Influence of polyethylene glycol 400 on the gastrointestinal absorption of ranitidine," Pharmaceutical Research, vol. 19, no. 9, pp. 1368-1374, 2002.

[41] S. C. Boca and S. Astilean, "Detoxification of gold nanorods by conjugation with thiolated poly(ethylene glycol) and their assessment as SERS-active carriers of Raman tags," Nanotechnology, vol. 21, no. 23, Article ID 235601, 2010.

[42] T. Niidome, M. Yamagata, Y. Okamoto et al., "PEG-modified gold nanorods with a stealth character for in vivo applications," Journal of Controlled Release, vol. 114, no. 3, pp. 343347, 2006.

[43] J. Kneipp, H. Kneipp, B. Wittig, and K. Kneipp, "Novel optical nanosensors for probing and imaging live cells," Nanomedicine, vol. 6, no. 2, pp. 214-226, 2010.

[44] J. Kneipp, H. Kneipp, M. McLaughlin, D. Brown, and K. Kneipp, "In vivo molecular probing of cellular compartments with gold nanoparticles and nanoaggregates," Nano Letters, vol. 6, no. 10, pp. 2225-2231, 2006.

[45] X. Qian, J. Li, and S. Nie, "Stimuli-responsive SERS nanoparticles: conformational control of plasmonic coupling and surface Raman enhancement," Journal of the American Chemical Society, vol. 131, no. 22, pp. 7540-7541, 2009.

[46] C. Matthäus, T. Chernenko, J. A. Newmark, C. M. Warner, and M. Diem, "Label-free detection of mitochondrial distribution in cells by nonresonant Raman microspectroscopy," Biophysical Journal, vol. 93, no. 2, pp. 668-673, 2007. 

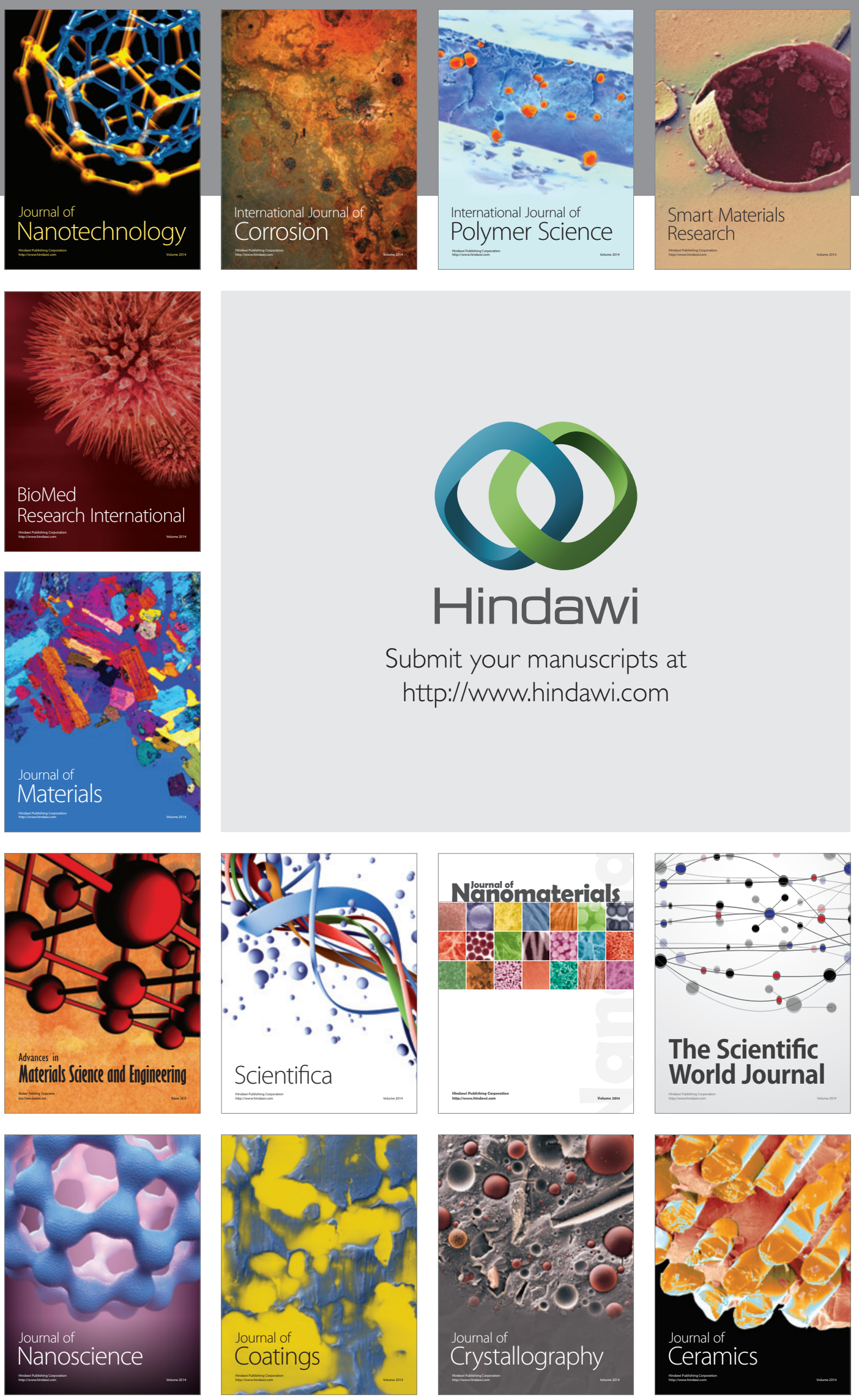

The Scientific World Journal

Submit your manuscripts at

http://www.hindawi.com

\section{World Journal}

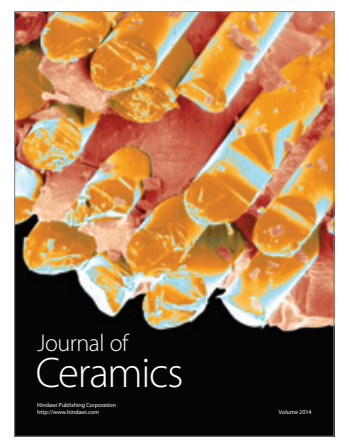

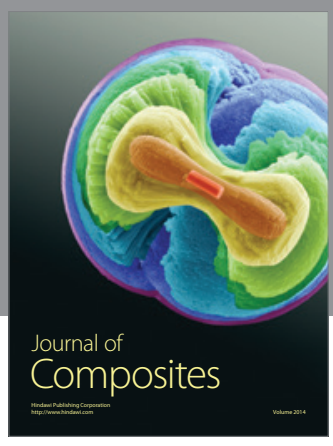
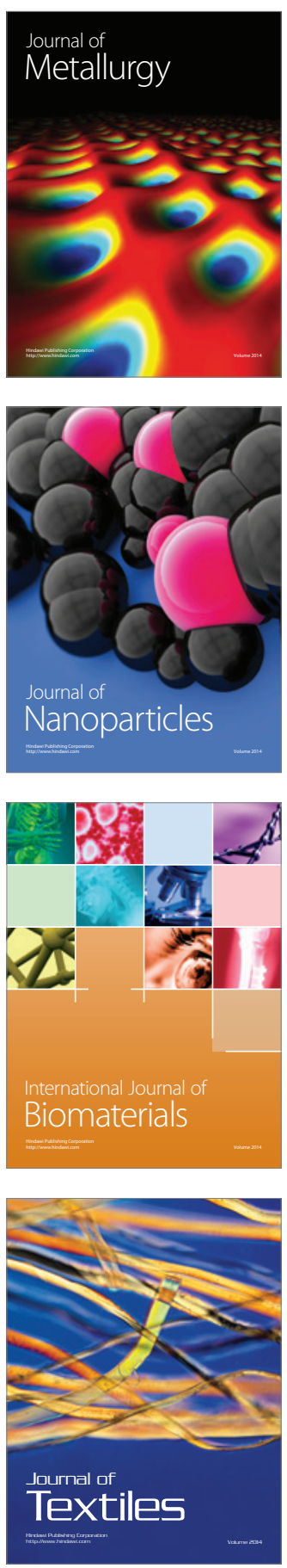